\title{
Philosophiques
}

\section{La nouvelle casuistique et la naturalisation des normes}

\section{Jean-Yves Goffi}

Volume 28, numéro 1, printemps 2001

La nature des normes

URI : https://id.erudit.org/iderudit/004929ar

DOI : https://doi.org/10.7202/004929ar

Aller au sommaire du numéro

Éditeur(s)

Société de philosophie du Québec

ISSN

0316-2923 (imprimé)

1492-1391 (numérique)

Découvrir la revue

Citer cet article

Goffi, J.Y. (2001). La nouvelle casuistique et la naturalisation des normes. Philosophiques, 28(1), 87-107. https://doi.org/10.7202/004929ar

\section{Résumé de l'article}

Il s'agit de déterminer le statut des normes chez les nouveaux casuistes (A. Jonsen, S. Toulmin, R.B. Miller). On indique d'abord quelles sont les circonstances de cette nouvelle casuistique ; on suggère ensuite qu'elle exporte dans le domaine de l'éthique certaines thèses de T. Kuhn, comme le " modèle déductiviste ", qu'elle prétend détrôner, s'alimentait au schéma Hempel-Oppenheim de l'explication scientifique. On suggère enfin que les nouveaux casuistes procèdent, de façon plus ou moins radicale, à une naturalisation des normes.
Ce document est protégé par la loi sur le droit d'auteur. L'utilisation des services d'Érudit (y compris la reproduction) est assujettie à sa politique d'utilisation que vous pouvez consulter en ligne.

https://apropos.erudit.org/fr/usagers/politique-dutilisation/ 


\title{
La nouvelle casuistique et la naturalisation des normes
}

\author{
JEAN-YVES GOFFI \\ Université de Grenoble \\ Jean-Yves.Goffi@upmf-grenoble
}

RÉSUMÉ. - Il s'agit de déterminer le statut des normes chez les nouveaux casuistes (A. Jonsen, S. Toulmin, R.B. Miller). On indique d'abord quelles sont les circonstances de cette nouvelle casuistique ; on suggère ensuite qu'elle exporte dans le domaine de l'éthique certaines thèses de T. Kuhn, comme le « modèle déductiviste », qu'elle prétend détrôner, s'alimentait au schéma Hempel-Oppenheim de l'explication scientifique. On suggère enfin que les nouveaux casuistes procèdent, de façon plus ou moins radicale, à une naturalisation des normes.

ABSTRACT. - It is intented to investigate about the status of norms in the thought of the new casuists (A.Jonsen, S. Toulmin, R.B. Miller). First, the circumstances of the new casuistry are set out; then it is suggested that the new casuists have integrated in their argument concepts from T. Kuhn, just as the "deductivist model" they so sharply criticize integrates concepts from C.G. Hempel and P. Oppenheim. With regard to the initial question, it is shown that the new casuists uphold a kind of norms-naturalizing constructivism.

Une note de l'article de S. Darwall, A. Gibbard et P. Railton, « Toward Fin de siècle Ethics : Some Trends ", ouvre une porte par laquelle je voudrais, en quelque sorte, me glisser. Ses auteurs, pour s'excuser de ne pouvoir leur accorder qu'une attention cursive ou de devoir les négliger, y indiquent toutes sortes de tendances caractéristiques de la philosophie morale contemporaine : nouvelles moutures du kantisme, de l'éthique de la vertu et du conséquentialisme; renouveau des études savantes portant sur l'histoire de l'éthique ; développement de l'éthique appliquée ; critiques féministes de la philosophie morale dominante, etc. Ils ne mentionnent pas dans cette liste l'intérêt croissant manifesté à l'égard de la casuistique. Il est vrai qu'un tel intérêt, pouvant aller jusqu'à la réhabilitation, est assez paradoxal puisqu'au même moment beaucoup de théologiens en dénoncent les insuffisances et les limites (individualisme, formalisme, légalisme, etc.). Il est vrai également que le livre pionnier en ce domaine est sous-titré par ses auteurs : A History of Moral Reasoning ${ }^{1}$, auquel cas il serait compris dans la rubrique «Ouvrages

1. Jonsen, Albert. R. et Toulmin, Stephen, The Abuse of Casuistry. A History of Moral Reasoning, Berkeley et Los Angeles, University of California Press, 1988. Bien entendu, la casuistique n'a jamais cessé d'être pratiquée, par exemple dans la formation des prêtres ou des rabbins, et la redécouverte de Jonsen et Toulmin n'est pas une résurrection : leur ouvrage est cependant pionnier en ce qu'il envisage la casuistique selon une perspective qui n'est pas celle de la théologie morale ou pastorale.

PHILOSOPHIQUES 28/1 — Printemps 2001, p. 87-107 


\section{8 - Philosophiques / Printemps 2001}

consacrés à l'histoire de l'éthique ». Cependant, si certains philosophes moraux contemporains manifestent un intérêt accru pour la casuistique, ce n'est sans doute pas seulement en vertu d'un surcroît d'attention porté à l'histoire du raisonnement moral. Comme il ne s'agit pas non plus de ministres du culte en charge d'âmes ou ayant pour mission d'administrer le sacrement de pénitence, ils voient plutôt en elle une méthodologie susceptible d'éclaircir la nature du raisonnement pratique. En ce sens, leur démarche s'inscrit dans le droit fil de cette "grande expansion " qui, à partir des années 50 modifia la donne en philosophie morale. Car c'est peu de dire que la situation antérieure était caractérisée par la prédominance, en métaéthique d'une orthodoxie non-cognitiviste ; elle était plutôt caractérisée par la prédominance de la métaéthique. C.D. Broad, pour ne citer que lui, avait depuis longtemps exposé les conséquences de cet état de fait :

En appeler à la théorie éthique de l'action correcte ne pourra pas plus nous enseigner à agir de façon correcte qu'en appeler à la théorie mathématique de la trajectoire de la balle de golf ne nous rendra capables de jouer au golf. De la sorte, l'intérêt de l'éthique est presqu'exclusivement théorique, comme ce serait le cas pour une théorie mathématique du golf ou du billard. ${ }^{2}$

S'il est vrai que la théorie éthique est, par définition, une activité qui s'exerce dans le domaine de la théorie, il est pour le moins paradoxal de voir en l'éthique à proprement parler une discipline dont l'intérêt est presqu'exclusivement théorique. La tradition la tient, au contraire, pour une discipline pratique et c'est une posture à la Broad qui est profondément atypique. Pour filer jusqu'au bout la métaphore, ce que désirent la plupart de ceux qui pratiquent le golf ou le billard, c'est de parvenir à un niveau au moins honorable et, si possible, plus qu'honorable, au golf ou au billard. Dans ces conditions, la casuistique, comme étude raisonnée de cas moralement problématiques, apparaît comme l'analogue de l'entraînement qui fait de quelqu'un, une fois acquis les rudiments, un joueur convenable : certainement pas le tout de l'éthique, mais une partie importante de celle-ci. En ce sens, l'intérêt pour la casuistique est l'indice du retour en force des questions normatives et substantielles; il rappelle qu'on est en droit d'attendre de l'éthique une réflexion sur des situations concrètes, menée à des fins d'évaluation et de décision.

Ceci étant, cette redécouverte de la casuistique ne se fait pas sans présupposés ni sans intentions. Je voudrais montrer comment certaines réappropriations influentes de celle-ci expriment une variété de constructivisme dans le domaine des normes morales. Je procéderai en trois moments dont chacun des points essentiels sera précisé au fur et à mesure.

2. Broad, Charlie D., Five types of Ethical Theory, Londres, Kegan Paul, Trench \& Trubner, 1930, p. 285. 
Je me demanderai, en premier lieu, ce que sont les circonstances de la casuistique contemporaine, par rapport aux circonstances de la casuistique antérieure. Il s'agira de montrer, en m'en tenant au plus près des intentions de ceux qui ont tenté de réhabiliter la casuistique, qu'ils s'y sont essayé dans un contexte d'insatisfaction face à la posture analytique adoptée en philosophie morale au cours de la première moitié de ce siècle ; et que leur tentative est solidaire d'une évaluation particulière des fonctions de l'éthique. Par " circonstances de la casuistique ", je n'entends rien que de très connu. D. Hume reconduit l'origine de cette vertu artificielle qu'est la justice à deux types de circonstances, c'est-à-dire de conditions particulières. Les unes sont propres à la nature humaine (égoïsme ; générosité limitée) ; les autres sont propres aux circonstances extérieures (rareté des objets par rapport aux besoins ; facilité de ces mêmes objets à changer de mains). A la suite de V. MunozDardé ${ }^{3}$ qui appelle ce complexe de données circonstances de la justice, j'appellerai circonstances de la casuistique le complexe de données qui est à l'origine de la casuistique.

Bien conscient de simplifier à l'extrême une question extrêmement difficile, je me risquerai à suggérer les circonstances suivantes de la casuistique classique. Comme le remarque A.R. Jonsen ${ }^{4}$, les religions du Livre, outre qu'elles comportent un enseignement moral, ont la particularité de formuler leurs principes moraux en termes de commandements universels. Le fidèle sait qu'il doit agir, en toutes circonstances, selon la loi de Dieu. Mais les circonstances, précisément, peuvent être à ce point enchevêtrées qu'il est parfois extrêmement difficile de discerner ce que commande la loi. Lorsque la complexité des situations singulières fait que leur application ne va pas de soi, se pose alors toute une série de questions relatives à l'interprétation qu'il convient de donner aux commandements divins. Pour prendre un exemple massif, même un commandement aussi indiscutable que : "Tu ne tueras pas " doit être interprété. Concerne-t-il la seule vie humaine ou bien également la vie animale ou même végétale ? Comment doit-il être interprété lorsqu'on a affaire à un injuste agresseur ? à un assassin qui manifeste un repentir sincère à l'issue d'un procès équitable ? Et ainsi de suite. L'idée sous-jacente est que plus s'accroît la distance entre l'universalité de la loi et la singularité des cas concrets, plus se fait sentir le besoin d'une casuistique.

Par ailleurs, la casuistique classique est, le plus souvent, une pratique de directeur de conscience ou de confesseur. Or, l'administration du sacrement de pénitence comporte la confession et l'absolution des péchés. Mais

3. Munoz-Dardé, Véronique, La justice sociale. Le libéralisme égalitaire de John Rawls, Paris, Nathan-Université, 2000, p. 7-8. De son côté, R.B. Miller intitule le premier chapitre de son livre Casuistry and Modern Ethics. A Poetics of Practical Reasoning, Chicago, The University of Chicago Press, 1996 : "The Occasions of Casuistry ». Dans ses traductions de J. Rawls, C. Audard rend circumstances of justice par « contexte de la justice ».

4. Jonsen, Albert R., " Casuistry ", dans Macquarie, John \& Childress, James, dir., A New Dictionary of Christian Ethics, Londres, SCM Press, 1986, p. 78. 


\section{Philosophiques / Printemps 2001}

un confesseur ne peut absoudre que s'il est capable de dire quels actes sont des péchés et à quel degré ils le sont, c'est-à-dire s'il est capable de les qualifier. C'est à dessein que j'emploie ce terme en provenance du vocabulaire juridique, les manuels post-tridentins comparant souvent le confesseur à un juge qui doit rendre une sentence après avoir étudié minutieusement une affaire ${ }^{5}$. Le fait qu'elle dépende d'une éthique des commandements divins; le fait qu'elle se rattache au sacrement de la confession : telles sont donc les circonstances ou les occasions principales de la casuistique ancienne.

Ceux qui, de nos jours, ont manifesté un regain d'intérêt pour la casuisitique en ont exposé les circonstances de façon assez détaillée. C'est par exemple, chez S.Toulmin, une évidente insatisfaction devant l'orientation prise par la philosophie morale depuis le «virage linguistique » opéré par G.E. Moore. En témoigne un article assez polémique de 1973 dans lequel notre auteur note le gouffre séparant les philosophes professionnels, exclusivement occupés de métaéthique, conçue comme une activité réflexive sur la nature même des questions et des jugements que l'on peut à bon droit qualifier de moraux, et les profanes, désespérément en quête d'avis éclairés relatifs à des questions substantielles. Mais $\mathrm{S}$. Toulmin observe également que, au cours de la période récente, lorsque des problèmes substantiels étaient effectivement abordés, hors du milieu universitaire, le débat prenait presque toujours la forme d'un affrontement entre des dogmatiques, se référant à un code de règles universelles ou à l'autorité d'un système religieux, et des relativistes ou des subjectivistes ${ }^{6}$, défendant une sorte de pluralisme en éthique sur la base de considérations relatives à la diversité des cultures et des attitudes. Au total, la situation semblait tout à fait bloquée pour ceux qui, comme lui, estimaient possibles la résolution rationnelle des désaccords en morale.

Ce n'est pas tant la justesse de ce diagnostic qui retiendra l'attention que ses implications. S. Toulmin semble considérer, en effet, qu'une telle situation a quelque chose de scandaleux en ce qu'elle constitue la négation même de cette entreprise collective que l'on nomme "éthique ". Ainsi, il avait affirmé, dès 1950 : «La fonction de l'éthique est de réconcilier les buts et les vouloirs indépendants d'une communauté humaine ${ }^{7}$.

Cette formule apparaît en fait comme la conséquence lointaine d'une thèse en métaéthique : la raison pour laquelle nous qualifions un jugement d'éthique est « le fait qu'il est employé afin d'harmoniser les actions des gens (plutôt que, par exemple, pour donner une description reconnaissable d'un

5. Mahoney, John, The Making of Moral Theology. A Study of the Roman Catholic Tradition, Oxford, Clarendon Press, 1987, p.24.

6. Ces termes n'ont pas ici leur signification technique et ne renvoient pas, en particulier, à la distinction classique selon laquelle le relativisme est une thèse morale (appartenant à l'éthique normative) et le subjectivisme une thèse éthique (appartenant à la métaéthique).

7. Toulmin, S., An Examination of the Place of Reason in Ethics, Cambridge, Cambridge University Press, 1950, p. 170. 
état de choses) ${ }^{8}$. Je laisse de côté les analyses, assez complexes, qui justifient une telle affirmation et me contente de préciser ce qu'elle signifie. Le point essentiel consiste en un parallèle entre les jugements scientifiques et les jugements moraux : tous les deux sont susceptibles de modifier quelque chose chez ceux qui les comprennent, c'est-à-dire qui comprennent quelles bonnes raisons viennent les étayer. Mais, dans le premier cas, ce qui est modifié c'est une série d'attentes ${ }^{9}$ tandis que, dans le second cas, ce qui est modifié c'est un ensemble de sentiments et de comportements. Dans ces conditions, la fonction de l'éthique, énoncée de façon plus détaillée sera la suivante : "Corréler nos sentiments et nos comportements de façon à rendre compatible, autant que faire se peut, la réalisation des buts et des désirs de chacun. " ${ }^{10}$.

Etant entendu que l'éthique est une entreprise où l'on cherche la satisfaction harmonieuse des désirs et des intérêts ${ }^{11}$, on comprend alors qu'une fonction essentielle du langage de la morale soit de permettre la correction de ce qui est spontanément éprouvé. Mais cette façon de concevoir les choses implique une évaluation bien particulière de la nature du désaccord en morale : celui-ci ne signale pas l'échec de la théorie, ni l'incompatibilité entre des principes irréconciliables. Il est plutôt la matière même sur laquelle l'éthique doit opérer, au moins initialement.

Cependant, il y a conflit et conflit. A l'origine, S. Toulmin pensait essentiellement aux conflits entre des intérêts et des buts. Il s'agit là de conflits constitutifs de l'éthique elle-même : dans un monde où existerait une sorte d'harmonie préétablie entre les intérêts des agents, l'activité que l'on appelle l'éthique serait sans raison d'être. Mais une fois celle-ci constituée, le conflit vient encore s'y manifester, cette fois-ci de l'intérieur. C'est dans le livre cosigné avec A.R. Jonsen que les choses vont être précisées. En effet, que l'on ait affaire à une démarche qui met en avant des concepts comme le devoir, la loi morale et les impératifs; ou à une démarche qui privilégie des concepts comme ceux de bien, de fins à atteindre ou de valeurs à réaliser, il est difficilement imaginable que les impératifs dans le premier cas ou les valeurs dans le second soient toujours parfaitement ajustés les uns aux autres. C'est d'ailleurs le cas même pour une pensée radicalement hostile à toute forme de légalisme. Après tout, le fameux jeune homme venu demander conseil à J.P. Sartre et que ce dernier renvoie à sa liberté, tout en sachant, par une espèce de science moyenne ce que sera, en définitive, son choix, vient bien le consulter parce qu'il a l'impression d'avoir rencontré un conflit entre des valeurs (rejoindre les FFL et oeuvrer pour la liberté et la démocratie ; rester auprès

8. Ibid., p. 145 .

9. En vertu de l'aphorisme selon lequel la fonction de la science est de "corréler nos expériences de telle façon que nous sachions ce à quoi nous aurons affaire ", Ibid., p. 125.

10. Ibid., p. 137.

11. La formule est à prendre au sérieux puisque $S$. Toulmin va jusqu'à définir les communautés comme des "groupes de gens vivant ensemble tout en respectant mutuellement leurs intérêts ", ibid, p. 135. 


\section{Philosophiques / Printemps 2001}

de sa mère et oeuvrer pour le soulagement d'une souffrance individuelle). Une occasion de la casuistique est donc le conflit moral, où la question consiste à classer des obligations ou des biens qui se font concurrence pour guider une conduite.

Mais, à supposer même que l'on se trouve dans une situation où un tel conflit ne se manifeste pas, des cas de conscience peuvent encore se rencontrer. En effet, certaines situations ressemblent à des cas d'école : Les obligations et les impératifs des éthiques déontologiques les concernent sans discussion possible ; ou bien, selon une autre interprétation, on y discerne immédiatement quels biens des éthiques téléologiques sont à promouvoir, et comment. Mais d'autres sont beaucoup plus difficiles à saisir : il semble que l'on ait du mal à les rattacher à une principe moral, même reconnu et avéré, par rapport auquel elles semblent marginales. Pour reprendre, en le modifiant, un exemple suggéré par A.R. Jonsen et $S$. Toulmin, une éthique déontologique du respect de la dignité de l'être humain et une éthique conséquentialiste de la promotion des intérêts des êtres dotés de sensibilité pourraient condamner également la cruauté envers les mammifères adultes : la seconde pour des raisons directes, une telle pratique lésant à l'évidence les intérêts d'êtres sensibles; la première pour des raisons indirectes, une telle pratique pouvant endurcir ceux qui s'y livrent et les disposer à traiter ensuite de façon indigne des êtres humains. Mais comment apprécieraient-elles la conduite d'un enfant qui prélève dans un étang de l'eau contenant des oeufs de grenouilles ?

Outre A. Jonsen et S.Toulmin, R.B. Miller est un de ceux qui ont oeuvré dans le sens d'une réhabilitation de la casuistique. Il se livre, pour son propre compte, à une analyse très proche de celle qui vient d'être résumée ${ }^{12}$. On notera toutefois certaines différences significatives. En premier lieu, il semble penser que les circonstances de la casuistique transcendent, d'une certaine façon, la situation contemporaine. C'est pourquoi on le voit, à propos de conflits moraux ou d'ambiguité, donner des références tirées aussi bien de contemporains que de médiévaux ou de penseurs de l'Antiquité ${ }^{13}$. En second lieu, il met, plus que A. Jonsen et S.Toulmin, l'accent sur le rôle que la question de la responsabilité ou de l'imputation a joué dans la constitution de la pensée casuistique : "Les expériences de l'ambiguiité, du conflit et de la responsabilité sont à l'origine de nombreux problèmes pour la délibération de style casuistique ${ }^{14}$.

En dernier lieu, il considère que chacune des occasions de la casuistique engendre, en quelque sorte, un élément de la pensée proprement casuistique. Parce qu'elle naît dans les situations de conflit moral, elle a développé une pensée qui procède par référence à des paradigmes. Parce qu'elle naît dans les

12. Miller, Casuistry and Modern Ethics. A Poetics of Practical Reasoning, Chap. I.

13. Ainsi, en ce qui concerne la question du mensonge : Cicéron, M. Walzer et Saint Augustin.

14. Ibid, p. 37. 
situations d'ambiguiité, elle a développé un type de pensée qui repose sur la présomption. Parce qu'elle naît lorsqu'il s'agit de déterminer le degré de responsabilité d'un agent moral par rapport à ses actions, elle a développé une théorie de l'acte. Je me suis donc employé à montrer que les circonstances de la nouvelle casuistique sont une conscience accrue de la complexité des situations où une décision éthique est requise, parallèle à une réticence avérée à recourir aux ressources des théories éthiques telles qu'elles sont envisagées dans la philosophie analytique.

Par ailleurs, les nouveaux casuistes opposent leur démarche, fondée sur l'analyse de cas, à une procédure déductiviste. Je voudrais montrer que cette procédure déductiviste présente une analogie structurelle avec une théorie bien connue de l'explication scientifique ; et que l'enjeu du débat consiste à savoir si l'on doit mettre l'accent sur la force normative du précédent (ou du paradigme) ou bien sur la force normative du principe. S. Toulmin qui, dans son livre de 1950, n'hésitait pas à comparer la résolution d'un problème moral substantiel à la résolution d'un problème technique par un ingénieur a $\mathrm{pu}$, si j'ose dire, mettre ses propres analyses au ban d'essai, en devenant, de 1975 à 1978, membre de la Commission Nationale pour la Protection des Sujets humains dans la Recherche Biomédicale et Comportementale. Cette Commission avait été constituée en 1974 par le Congrès des Etats-Unis et son principal objectif était de préciser les conditions qui rendaient moralement acceptables l'utilisation de sujets humains dans une recherche biomédicale ou comportementale. Il s'agissait donc de parvenir collégialement à une décision d'ordre pratique : rendre un avis concernant le caractère licite ou illicite d'un certain type d'action ${ }^{15}$.Or, selon S. Toulmin, la méthodologie adoptée par les membres de la Commission ne ressembla en rien à ce que l'on aurait pu prévoir, dans de telles circonstances, à la lumière des théories éthiques dominantes. Le terme "méthodologie » est d'ailleurs quelque peu égarant dans la mesure où il suggère l'application consciente et réfléchie d'une procédure permettant d'atteindre infailliblement un objectif déterminé à l'avance. Il serait plus juste de parler de démarche spontanément adoptée, sans concertation préalable, par une pluralité d'individus, chacun adhérant, pour son propre compte, à un système particulier de valeurs et de normes éthiques. Cette démarche consistait à procéder non pas déductivement, mais par analyse de cas. C'est en ce sens qu'elle peut être considérée comme une casuistique.

Que faut-il entendre par « modèle déductiviste » ? Il s'agit d'un type de raisonnement pratique non seulement compatible avec les différentes versions du déontologisme et du conséquentialisme, mais encore impliqué par elles. D'un certain point de vue, le modèle déductiviste consiste en la transposition, dans le domaine de la philosophie morale, du modèle explicatif dit "des lois de couverture » en philosophie des sciences. Il ne s'agit, bien

15. Par exemple : des malades mentaux, des mineurs ou des prisonniers peuvent-ils faire l'objet d'une expérimentation comportementale? 


\section{Philosophiques / Printemps 2001}

entendu, que d'une analogie et il ne saurait être question de lui faire dire plus qu'elle ne peut, d'autant que ni A.R. Jonsen, ni S.Toulmin, ni R.B. Miller ne la reprennent explicitement à leur compte. La différence est d'ailleurs évidente : le modèle des lois de couverture concerne l'explication scientifique alors que le schéma déductiviste concerne l'évaluation d'un problème moral. Il existe cependant une ressemblance structurelle entre ces deux démarches : dans les deux cas, en effet, le concept de subsomption joue un rôle essentiel (subsumer, c'est considérer le fait comme l'application d'une loi, ou le particulier comme compris sous l'universel).

La présentation canonique du modèle explicatif par lois de couverture se trouve dans un article de 1948, cosigné par C.G. Hempel et P. Oppenheim ${ }^{16}$. Ces auteurs considèrent que dans toute explication scientifique figure un explanadum (énoncé qui doit être expliqué) et un explanans (ensemble d'énoncés capables d'expliquer). Dans l'explanans, on peut distinguer : des énoncés décrivant des conditions antécédentes et des énoncés exprimant des lois générales. Le schéma d'une explication est alors le suivant :

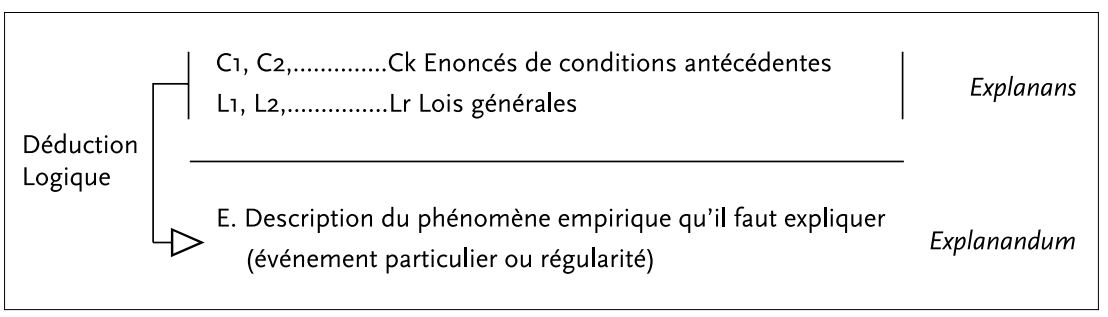

Il est crucial de noter que la relation entre l'explanans et l'explanandum est une relation de déduction logique. Les énoncés qui composent l'explanans et l'explanandum seront considérés respectivement comme les prémisses et comme la conclusion d'un raisonnement. Ce raisonnement sera valide si l'on ne rencontre jamais une situation où les prémisses seraient toutes vraies et la conclusion fausse. Pour C. G. Hempel, une grande partie du travail scientifique consiste à établir de façon précise les conditions antécédentes et à déterminer les lois constituant l'explanans. Le schéma, que l'on appelle parfois aussi nomologique-déductif, car la déduction n'a de valeur explicative que si elle comporte au moins une loi, est d'application universelle : il est censé valoir aussi bien pour les sciences physiques et pour les sciences biologiques que pour les sciences humaines (sous réserves de complications dues à l'existence de lois statistiques). Par conséquent, le schéma général que suit toute explication scientifique est le suivant ${ }^{17}$ :

16. Hempel, Carl Gustav et Paul Oppenheim, "Studies in the Logic of Explanation ", Philosophy of Science, 15, p.135-75.

17. Carnap, Rudolf, Les Fondements philosophiques de la physique, tr. fr. de J. M. Luccioni et A. Soulez, Paris, A.Colin, 1973 [1966], p. 15. 
1) $(\mathrm{x})(\mathrm{Px} \rightarrow \mathrm{Qx})$

2) $\mathrm{Pa}$

3) $\mathrm{Qa}$

1.dit de tout objet que, s'il possède la propriété $\mathrm{P}$, il possède alors la propriété Q : c'est la loi universelle. 2. dit de l'objet $a$ qu'il possède la propriété $\mathrm{P}$ : c'est la condition antécédente. 3 . dit de l'objet $a$ qu'il possède la propriété Q : c'est la conclusion, qui peut être inférée de 1 . et de 2 . Décrire de la sorte le schéma nomologique-déductif permet de faire de l'énoncé qui exprime la loi universelle la majeure, et de l'énoncé qui exprime la relation antécédente, la mineure, d'un syllogisme concluant.

Un exemple élémentaire permettra de comprendre comment fonctionne ce modèle. Soit à expliquer que lorsqu'un thermomètre est plongé dans de l'eau chaude on constate, en un premier temps, une légère baisse du niveau de la colonne de mercure, suivie d'une élévation de ce niveau. On expliquera les choses ainsi : en premier lieu le verre, mais pas le mercure qu'il contient, se dilate sous l'effet de la chaleur, d'où la baisse du niveau; en second lieu, le mercure lui-même se dilate, d'où l'élévation du niveau. Ce type d'explication est bien suffisant dans un contexte familier. Mais on pourra se conformer au schéma Hempel-Oppenheim : il n'apporte rien d'inédit en ce qui concerne le contenu de l'explication elle-même ; il présente simplement les choses avec un surcroît de rigueur. On mettra alors en évidence un certain nombre de conditions antécédentes, par exemple :

- $\quad$ C1 : le tube est en verre.

- C2 : le tube est partiellement rempli de mercure.

- Cn : le tube est immergé dans de l'eau chaude.

De même, on se référera à des lois ou à des énoncés généraux pertinents dans ce contexte; par exemple :

- $\quad$ L1 : Loi de dilatation thermique du verre.

- L2 : Loi de dilatation thermique du mercure.

- Ln : Faible conductivité thermique du verre.

Le phénomène décrit apparaîtra alors comme la conclusion, d'un raisonnement dont les prémisses seraient les énoncés C1, C2 ... Cn d'une part, et L1, L2... Ln d'autre part. On peut noter, à propos du schéma HempelOppenheim, que les lois elles-mêmes peuvent être expliquées par subsomption sous des lois plus générales. Ainsi, la loi galiléenne pour les corps en chute libre peut être expliquée par déduction à partir des lois newtoniennes du mouvement et de la loi newtonienne de la gravitation. Le schéma HempelOppenheim, en un mot, suggère une hiérarchie : les faits, les lois qui permettent de les expliquer (toujours de forme universelle, elles peuvent être plus ou moins générales), enfin, les théories, c'est-à-dire des systèmes d'énoncés très généraux comportant des concepts qui réfèrent à des entités non-observables, mais postulées à des fins d'explication des régularités observées. On retrouvera, mutatis mutandis, la même structure « de haut en bas » dans le modèle déductiviste du raisonnement éthique. Un exemple fera comprendre 


\section{Philosophiques / Printemps 2001}

ce dont il s'agit ${ }^{18}$. Soit un jugement éthique de niveau moyen à propos d'une expérimentation médicale sans bénéfice thérapeutique direct, menée sur de très jeunes enfants. Il pourra prendre la forme suivante :

- Prémisse normative : On ne doit jamais utiliser une personne à titre de sujet dans une expérimentation médicale sans bénéfice thérapeutique direct à moins qu'elle n'ait donné un consentement libre et éclairé à sa participation à cette expérimentation.

- Prémisse descriptive : Les très jeunes enfants sont des personnes incapables de donner leur consentement libre et éclairé à une participation comme sujets dans le cadre d'une expérimentation médicale sans bénéfice thérapeutique direct.

- Conclusion: On ne doit jamais utiliser de très jeunes enfants à titre de sujets dans une expérimentation médicale sans bénéfice thérapeutique direct.

Nous avons dit que ce jugement est de niveau moyen : concrètement, cela signifie que sa conclusion (normative) peut devenir à son tour la prémisse (normative) d'un jugement plus spécifique ; ainsi :

- Prémisse normative: On ne doit jamais utiliser de très jeunes enfants à titre de sujets dans une expérimentation médicale sans bénéfice thérapeutique direct.

- Prémisse descriptive : Le protocole du Docteur Dupont, relatif à la surveillance des modifications du transit intestinal en cas de traitement par photothérapie de l'hyperbilirubinémie non conjuguée du prématuré implique l'utilisation de très jeunes enfants dans une expérimentation médicale sans bénéfice thérapeutique direct.

- Conclusion : Le protocole du Docteur Dupont doit être refusé.

Mais cela signifie également que la prémisse (normative) du jugement initial peut être considérée comme la conclusion (normative) d'un jugement plus général ; ainsi :

- Prémisse normative : Une personne ne doit jamais être traitée comme un moyen seulement, mais toujours en même temps comme un fin.

- Prémisse descriptive : Utiliser une personne à titre de sujet dans une expérimentation médicale sans bénéfice thérapeutique direct sans avoir obtenu au préalable son consentement libre et éclairé, c'est la traiter comme un moyen seulement.

- Conclusion normative : On ne doit jamais utiliser une personne à titre de sujet dans une expérimentation médicale sans bénéfice thérapeutique direct à moins qu'elle n'ait donné un consentement libre et éclairé à sa participation à cette expérimentation.

L'analogie avec le modèle nomologique-déductif est certaine (même si, rappelons le, dans un cas il s'agit d'expliquer un fait et dans l'autre de justifier

18. Je l'emprunte, pour partie à Graber, Glenn C. et Thomasma, David, C., Theory and Practice in Medical Ethics, New York, Continuum Publishing Company, 1989. 
son assentiment à un jugement moral). Mais l'exemple qui précède permet de comprendre comment on "descend " d'un énoncé normatif très général (principe du respect de l'autonomie de la personne) jusqu'à un énoncé normatif très spécifique, de même que l'on pouvait « descendre " d'une loi très générale (lois du mouvement de Newton, par exemple) jusqu'à une explication très spécifique. En outre, de même que dans le modèle nomologique-déductif la « force » explicative du raisonnement vient de la présence, dans les prémisses, d'une loi au moins, de même, dans l'approche déductiviste, la «force " normative du raisonnement vient de la présence, dans les prémisses, d'un énoncé normatif au moins. En fait, la «force " normative de la conclusion est fonction de la " force » normative de la majeure. Comme l'écrivent A. Jonsen et S. Toulmin : «La vérité ou la certitude (certainty) qui s'attache à ces axiomes est transmise du haut vers le bas jusqu'aux cas spécifiques qu'il faut "prouver». » ${ }^{19}$.

Etant bien entendu que la situation concrète est spécifiée, dans ce type de démarche, par la prémisse descriptive, le schéma qui vient d'être décrit permet toujours, en principe, d'aboutir à l'évaluation d'un telle situation lorsque la prémisse normative comporte des expressions telles que : «il est bien de... », " il est mal de... », « il est juste de.... » - c'est-à-dire lorsque la norme s'y présente comme une valeur - ; il permet, de la même façon, de parvenir à une décision relative à cette situation, lorsque la prémisse normative comporte des expressions telles que : «il faut... ; ", « il ne faut pas... », " on doit... », « on ne doit pas... »- c'est-à-dire lorsque la norme s’y présente comme une loi -. Un telle démarche est, bien entendu, compatible avec une certaine forme de casuistique. C'est le cas, par exemple, chez saint Thomas d'Aquin qui affirme d'une part que la loi de nature est identique pour tous selon ses premiers principes généraux, mais qu'elle peut comporter des exceptions lorsqu'on descend aux détails. La vérité des principes communs de la raison pratique est connue de tous. Mais ses conclusions propres en matière de préceptes seconds ne comportent pas nécessairement une vérité qui soit la même pour tous à cause de l'extrême complexité des situations singulières. En ce sens, la conscience n'est infaillible que lorsque la conclusion particulière tombe directement sous la prémisse normative universelle ${ }^{20}$. Si ce n'est pas le cas, une réflexion relevant de la casuistique devient alors nécessaire.

Toutefois, ce n'est pas à cela que pensent $\mathrm{A}$. Jonsen et $\mathrm{S}$. Toulmin et c'est pourquoi ce qu'ils nomment casuistique correspond à une démarche représentée par un schéma sensiblement différent. Nos auteurs rattachent le raisonnement moral à l'exercice de la prudence, en un sens aristotélicien.

19. Jonsen et Toulmin, The Abuse of Casuistry, p. 34. Ces auteurs empruntent à I. Lakatos la métaphore de la vérité ou de la certitude qui se transmettent dans un sens ou dans un autre.

20. Les analyses de saint Thomas d'Aquin entretiennent une certaine ambiguïté: lorsqu'on parvient aux situations singulières qui rendent nécessaire la mise en oeuvre d'une casuistique, la difficulté consiste-t-elle à appliquer une règle ou bien à déduire une règle à partir de principes premiers ? Sur ce point, on peut consulter de Potts, Timothy C., Conscience in Medieval Philosophy, Cambridge, Cambridge University Press, 1980, en particulier le chap. IV. 


\section{Philosophiques / Printemps 2001}

Rappelons que la prudence, ou sagesse pratique, est, chez le Stagirite, la vertu capable de réaliser la finalité générale de la vie humaine (soit le bonheur) dans les circonstances complexes et contingentes d'un monde sublunaire. Elle ne consiste pas à appliquer des normes universelles, mais est plutôt une intelligence des situations : elle porte sur les faits particulier ultimes qui ne sont pas objets de science, mais de perception. A. Jonsen et $S$. Toulmin interprètent les choses comme suit : les cas moralement problématiques, ceux à propos desquels il est nécessaire d'argumenter rationnellement, ne se rencontrent pas lorsqu'il est devenu difficile de subsumer un cas particulier sous une règle ou de déduire des préceptes seconds à partir de préceptes premiers, eux-mêmes déduits à partir de principes connus par soi. Il s'agit bien, certes, de cas complexes, litigieux, difficiles à traiter parce que se produisant dans des circonstances extrêmement enchevêtrées. Mais cet enchevêtrement n'a pas tant pour effet de dissimuler un principe ou une règle que de rendre difficile à saisir ce qui rattache le cas présent à un précédent, c'est-à-dire à un autre cas, luimême déjà établi et décidé. Cela revient à dire que le propre de la démarche casuistique consiste à évaluer le cas litigieux à la lumière d'un cas antérieur qui fait figure de solution déjà adoptée. On y a affaire à un type de raisonnement qui n'est pas déductif, mais jurisprudentiel. Pour dire les choses aussi simplement que possible : dans le droit romano-germanique, le juge statue en fonction d'un corps de règles préétablies qui sont le plus souvent d'expression législative. Dans les pays de Common-Law, au contraire, le juge statue en s'en tenant à la règle antérieurement dégagée (règle du stare decisis). Il résout l'affaire en regardant en arrière et en cherchant à savoir comment ses prédécesseurs ont résolu des affaires antérieures qui présentaient des faits similaires. C'est à ce type de raisonnement jurisprudentiel que se rattache la casuistique ; elle s'en démarque en ce que, pour elle, le précédent est un cas simple. A. Jonsen et S. Toulmin comme R.B. Miller lui assignent un statut de paradigme. Pour les premiers, le précédent est simple en ce sens que le cas paradigmatique illustre la violation directe d'un principe, pris en son sens le plus manifeste ${ }^{21}$; pour le second, le paradigme est simple en ce qu'il fournit un exemple dans lequel le vocabulaire moral et les procédures logiques sont relativement clairs et éprouvés ${ }^{22}$. Le mode de fonctionnement du raisonnement de type casuistique est alors le suivant : «Les vérités et les certitudes (certitudes) établies dans les cas précédents passent de côté (pass sideways) de telle sorte qu'elles permettent la "résolution » de problèmes ultérieurs " 23 .

L'idée est la suivante : parvenir à une évaluation ou à une décision d'ordre éthique requiert en premier lieu une description de la situation à propos de laquelle on doit se prononcer. Il s'agit de mettre en évidence les

21. Jonsen et Toulmin, The Abuse of Casuistry, p. 252. La formule a son symétrique : le paradigme peut aussi illustrer la conformité à un principe, pris en son sens le plus manifeste.

22. Miller, Casuistry and Modern Ethics, p. 155.

23. Jonsen et Toulmin, The Abuse of Casuistry, p. 35. 
"particularités du cas ${ }^{24}$. Cela permet de déterminer jusqu'à quel point le cas présent ressemble à ce paradigme qui a valeur de précédent et à propos duquel un consensus est établi. Le rapprochement avec le cas paradigmatique crée alors une présomption : ce qu'il était licite ou illicite de faire dans ce cas, il est sans doute licite ou illicite de le faire maintenant. Cette présomption ne vaut, cependant, que sous réserve que des éléments nouveaux ne soient pas mis en évidence, éléments qui pourraient brouiller la ressemblance avec le cas paradigmatique. Cette clause distingue encore le modèle casuistique du modèle jurisprudentiel ; A. Jonsen et S. Toulmin, à cause d'elle, le comparent au diagnostic médical. Au total, le schéma casuistique prendra la forme suivante :

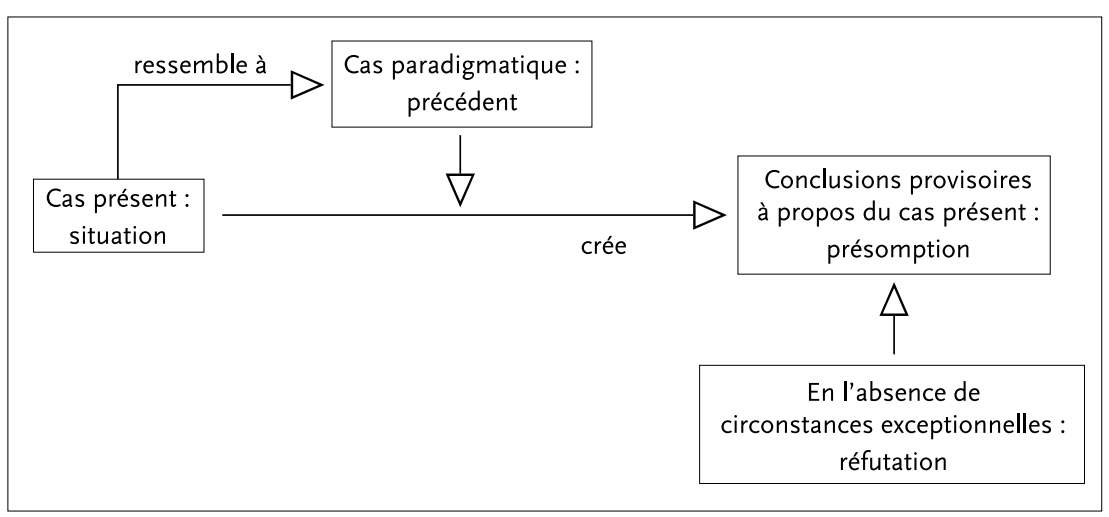

Un exemple très élémentaire montrera ce dont il s'agit. Chacun admet qu'on doit rendre, en bon état à son propriétaire, l'objet qu'on lui a emprunté, lorsqu'on n'en a plus l'usage. Il s'agit là, pourrait-on dire, du paradigme de la restitution. En l'absence de circonstances exceptionnelles, se conformer au paradigme résout toute contestation et permet de prendre une décision. Mais toutes sortes de circonstances peuvent venir compliquer les choses, comme dans le cas suivant :

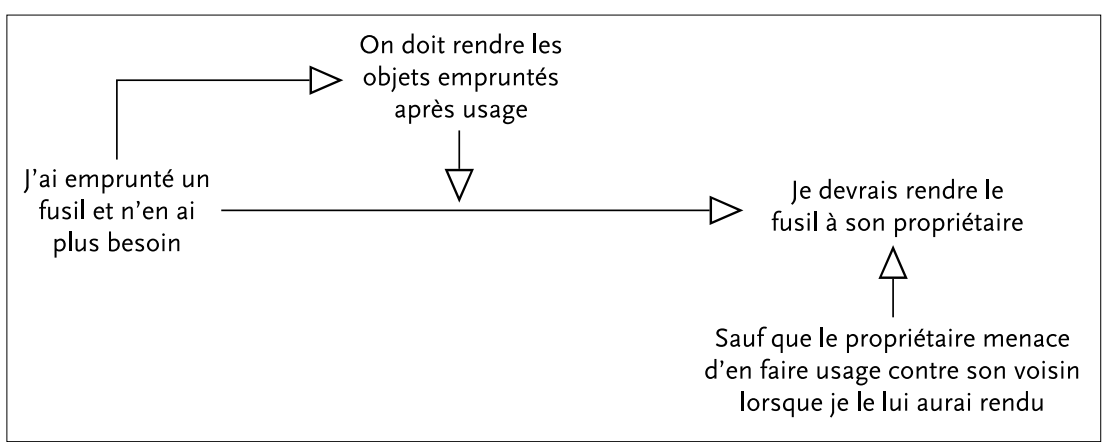

24. Il s'agit là d'une allusion à l'Éthique à Nicomaque, III, 2, 1111a, 1-22. 
On voit que la situation initiale peut être caractérisée par des circonstances infiniment variées : au lieu d'un fusil, l'objet emprunté peut être inoffensif, mais d'une valeur tellement infime que la règle de restitution ne s'applique peut-être pas ; ou bien l'objet emprunté peut appartenir, en fait à quelqu'un d'autre; ou bien il peut s'agir d'une pièce à conviction dans un procès criminel; ou bien il a pu être endommagé pendant qu'il était en ma possession ; ou bien il m'a été prêté endommagé, sans que je m'en aperçoive ; et ainsi de suite. Toutes ces circonstances peuvent contribuer à affaiblir la présomption initialement établie. C'est en ce sens particulier qu'elles constituent des réfutations. Ce mot traduit d'ailleurs l'anglais rebuttals, terme qui n'a certainement pas été choisi au hasard par A. Jonsen et S. Toulmin; en anglais juridique, en effet, le mot rebuttal désigne toute indication de preuve (evidence) susceptible de nier ou d'affaiblir une indication de preuve de la partie adverse.

Ce modèle «latéral » appelle plusieurs commentaires. En premier lieu, la force normative des conclusions auxquelles il permet d'aboutir est une fonction de la ressemblance entre le cas effectivement envisagé et le cas paradigmatique. Maximale dans les situations où le cas paradigmatique et le cas discuté présentent de nombreuses analogies, elle décroît dès lors que ces analogies sont moins saillantes; elle devient minimale dans les cas marginaux ou ambigus. On peut même imaginer qu'un cas litigieux soit, pour ainsi dire, à égale distance de deux cas paradigmatiques et qu'il soit, de la sorte, possible de la rattacher à l'un aussi bien qu'à l'autre. En second lieu, une telle façon de procéder suppose qu'on raisonne en regroupant les choses qui se ressemblent et en séparant celles qui diffèrent. Elle ne peut donc être mise en oeuvre que si l'on dispose d'une taxonomie, c'est-à-dire d'un inventaire raisonné, aussi précise et aussi complète que possible des cas paradigmatiques. En dernier lieu, il importe de noter que le modèle casuistique n'est pas contextualiste ou situationnaliste : ce n'est pas un modèle de décision au cas par cas où les parties concernées laisseraient jouer la dynamique du contexte ou de la situation jusqu'à ce qu'elle conduise à une évaluation (ou à une prise de décision) convenable. Les principes et les règles moraux ne sont pas abandonnés; on considère plutôt qu'il n'est ni nécessaire, ni éclairant de les penser à part de cas paradigmatiques. Il faut donc distinguer le fait de raisonner à partir de principes et le fait de raisonner en accord avec des principes.

Ce que contestent donc les partisans de la nouvelle casuistique, c'est essentiellement l'intérêt et la fécondité d'une sorte de géométrie morale qui consisterait à déployer les conséquences et les implications d'une théorie éthique afin de permettre une prise de décision relative à un problème donné. En ce sens, la comparaison de $S$. Toulmin entre la résolution d'un problème moral et d'un problème technique n'est pas aussi déplacée qu'on pourrait penser au premier abord. Cela ne signifie évidemment pas qu'un éthicien est une espèce d'ingénieur, expert en matière de jugement moral. Mais un ingénieur n'applique pas les théories physiques au sens où il déduirait une 
machine à partir de principes et de lois. Il en fait plutôt usage comme de guides pour l'action. Il en est vraisemblablement de même dans la casuistique.

Je voudrais maintenant indiquer que les nouveaux casuistes, en raison de l'accent mis par eux sur le précédent, et en raison de la conception qu'ils s'en font rencontrent une difficulté à laquelle les défenseurs de la casuistique «à l'ancienne » n'étaient pas confrontés ; et qu'ils y remédient en recourant à une sorte de constructivisme naturalisée. Pour commencer, je voudrais prendre un peu de recul par rapport au schéma proposé par les nouveaux casuistes. Indiscutablement, aux yeux de ceux qui l'ont proposé, il a une valeur descriptive : il donne une image fidèle de ce qui se passe dans une commission ou dans un comité d'éthique. Il montre également qu'en matière d'éthique, les entreprises d'évaluation ou de décision collective ne sont pas plus condamnées à prendre la forme d'un conflit entre opinions irréconciliables qu'à parvenir à la création d'un consensus honteux, par abandons successifs de convictions fondamentales. Pour ne pas affecter une forme strictement déductive à partir de principes premiers, la discussion n'est pas non plus vouée à prendre une forme irrationnelle. Au lieu de demander : "Sous quel principe le cas présent se subsume-til ? ", on va plutôt se demander : " Où le cas présent est-il localisé sur la carte des cas paradigmatiques ?". Mais parler de cas paradigmatiques n'est pas sans conséquences. Pour des raisons qu'il vaudrait certainement la peine de préciser, le terme paradigme est devenu, en effet, un mot à tout faire en philosophie : H. Jonas fait des parents et des hommes d'Etat les paradigmes éminents de la responsabilité ; les défenseurs radicaux de l'environnement mettent en cause, à travers une attitude arrogante envers la nature, un certain paradigme technocratique-anthropocentré ; on parle couramment de la nouvelle conception de la science qui se met en place à la fin de la Renaissance comme du paradigme Bacon-Descartes; et ainsi de suite. Pour leur part, nous l'avons vu, les partisans de la nouvelle casuistique considèrent à tout le moins les cas paradigmatiques comme des cas simples et non sujets à controverse. Pour cette raison, A. Jonsen et $\mathrm{S}$. Toulmin les assimilent à des eschata au sens d'Aristote, c'est-à-dire à des particuliers ultimes, dont la fonction en éthique est « d'être des objets ultimes d'appel et de comparaison en matière de délibération et de discussion ${ }^{25}$ ». Concrètement, cela signifie que si quelqu'un ne comprend pas en quoi la non restitution d'un objet emprunté et réclamé avec insistance par son propriétaire légitime est moralement condamnable, cela le disqualifie pour évaluer des cas plus délicats. Mais J.B. Miller emploie également le terme paradigme en un sens proche de celui que lui a conféré T. Kuhn : la casuistique, comme la science normale, est une pratique visant à résoudre des problèmes ; l'une et l'autre fournissent à une communauté de chercheurs un point de départ validé pour une recherche ultérieure ${ }^{26}$. Le rapprochement n'est pas gratuit, T. Kuhn lui-même comparant le paradigme scientifique au

25. Jonsen et Toulmin, The Abuse of Casuistry, p. 207.

26. Voir par exemple, Casuistry and Modern Ethics, p. 16. 
précédent juridique : "Comme une décision judiciaire admise dans le Droit Commun, c'est un concept destiné à être structuré et précisé dans des conditions nouvelles ou plus strictes. $»^{27}$

De même, la façon dont les nouveaux casuistes critiquent le modèle déductiviste semble inspirée de l'affirmation, par l'historien des sciences, de l'antériorité du paradigme par rapport aux règles. Mais je laisserai de côté la question de la pertinence de tous ces rapprochements, d'autant plus que R.B. Miller se montre assez prudent pour concéder que la casuistique et la science normale se ressemblent à bien des égards, mais pas sur tous les points. Elles se ressemblent assez, toutefois, pour qu'on ne puisse manquer de s'interroger : « Existe-t-il, dans le domaine de la morale, des événements comparables aux révolutions scientifiques? »; ou bien encore, de façon plus directe : "Existe-t-il, dans le domaine de la morale, des changements de paradigme ? ». A cette question, les auteurs qui nous intéressent vont répondre par un oui nuancé. Mais le caractère nuancé de la réponse ne doit pas masquer le fait qu'elle constitue une vraie rupture avec les analyses de la casuistique classique. Pour mettre ce point en évidence, je raisonnerai sur l'exemple de la prohibition augustinienne du mensonge. En un sens, cet exemple n'est pas tout à fait adéquat puisque saint Augustin écrit bien avant la constitution d'une casuistique au sens technique du terme. Cependant, les textes consacrés à la question du mensonge mettent bel et bien en place les éléments de ce que l'on pourrait appeler une protocasuistique. Le mensonge est défini par l'Evêque d'Hippone comme le fait d' "avoir une pensée dans l'esprit et, par paroles ou tout autre moyen d'expression, en énoncer une autre ${ }^{28}$. Quant au péché du menteur, il est défini comme le fait de « parler contre sa pensée avec l'intention de tromper ${ }^{29}$. Le mensonge est condamnable pour deux raisons. En premier lieu, il va contre la finalité même de la parole, qui n’a pas été « donnée aux hommes pour se tromper mutuellement mais pour porter leurs pensées à la connaissance d'autrui ${ }^{30}$. En second lieu, il va directement à l'encontre du commandement selon lequel on ne doit pas porter de faux témoignages. Ce commandement embrasse toutes les formes de mensonge puisque «en parlant, l'homme rend témoignage à son âme $»^{31}$. Par conséquent, le mensonge est absolument prohibé. Ceci étant, saint Augustin va distinguer plusieurs espèces de mensonge, plus ou moins pardonnables. Le mensonge commis dans l'enseignement de la religion est le plus détestable

27. Kuhn, T. S., La Structure des révolutions scientifiques, Paris, Flammarion, 1972, p. 39 [1970]. La traduction française donne : "droit commun " pour Common Law. J'ai pris la liberté de rétablir des majuscules, conformément à l’usage des juristes.

28. Augustinus, Aurelius, Du mensonge, III, 3.

29. Ibid., III, 3. Voir aussi Enchiridion, VII, 22. La clause de l'intention soulève des problèmes délicats, que je laisse de côté ici.

30. Enchiridion, VII, 22.

31. Du mensonge, V, 6. L'idée sous-jacente est qu'en parlant contre sa pensée, l'homme rend un faux témoignage à son âme. 
de tous ; le mensonge qui, sans faire de tort à personne, préserve quelqu'un d'un attentat impur est le plus excusable de tous. On peut aller jusqu'à dire de quelqu'un qui ne mentait que pour nuire et qui ne ment plus maintenant que pour le bien des autres qu'il a progressé. Il n'en reste pas moins qu' " autre chose est un fait louable en lui-même et autre chose un fait que l'on trouve préférable à un autre plus mauvais ${ }^{32}$. La morale de cette histoire est limpide : en distinguant plusieurs espèces de mensonge, saint Augustin rend possible une évaluation de type casuistique. Mais celle-ci ne saurait avoir pour fonction que de mesurer les écarts par rapport à la norme, qui reste la prohibition inconditionnelle du mensonge, et d'évaluer la gravité de sa transgression éventuelle. Il ne saurait être question d'admettre que la norme ellemême soit modifiée en quelque façon que ce soit au cours de cette opération. C'est la raison pour laquelle Pascal se scandalisera de ce dévoiement de la casuistique par lequel on imagine des stratagèmes (équivoques, restrictions mentales) permettant de se soustraire à la prohibition pesant contre le mensonge ${ }^{33}$. Les nouveaux casuistes, pour leur part, se démarquent de cette façon de procéder. Chez A. Jonsen et $\mathrm{S}$. Toulmin, les choses se passent ainsi : La casuistique comme pratique permettant d'évaluer et de résoudre des cas litigieux comporte ses propres difficultés : il n'est pas toujours simple de dire à quel paradigme on doit rattacher le cas présent ; on ne sait pas toujours quelle importance donner aux exceptions, etc. . Pour nos auteurs, ce ne sont pas nécessairement les individus qui seront aptes à déterminer des solutions dans ces cas difficiles. De telles solutions seront plutôt le produit de "processus historiques sur le long terme, incorporant une expérience collective ${ }^{34}$. Cela revient à faire de la casuistique une pratique réfléchie venant s'enter sur des pratiques spontanées ; d'ailleurs, A. Jonsen et S. Toulmin disent eux-mêmes du discernement, lequel constitue le noyau dur de la casuistique, qu'il n'est pas tant une aptitude à saisir les relations abstraites qu'une capacité « à construire une réflexion relative aux leçons pratiques de l'expérience concrète " ${ }^{35}$. Présenter les choses ainsi implique, à l'évidence, que les paradigmes ne sont pas donnés mais s'élaborent et se construisent, la théorie morale proprement dite jouant d'ailleurs un rôle assez modeste au cours de cette élaboration. Il s'agit là, à n'en pas douter, d'un approfondissement des tendances communautaristes révélées par S. Toulmin dans son livre de $1950^{36}$. Cette construction prend deux aspects : d'une part, le paradigme manifeste une certaine plasticité par rapport aux cas qui s'y rattachent, ces derniers pouvant être une source d'élucidation et de clarification au sens qu'il convient de donner aux cas typiques. D'autre part, les paradigmes eux-mêmes

32. Du mensonge, $\mathrm{V}, 7$.

33. Pascal, Blaise, Oeuvres Complètes, Paris, Seuil, 1963, p. 411-12.

34. Jonsen et Toulmin, The Abuse of Casuistry, p. 314.

35. Jonsen et Toulmin, The Abuse of Casuistry, p. 329.

36. Voir, par exemple, la note 11 supra. 


\section{Philosophiques / Printemps 2001}

peuvent, à l'occasion, être mis en cause de façon plus radicale encore : c'est ce qui se passe lorsque " la structure factuelle du paradigme est, en quelque façon, détruite ${ }^{37}$ ". On pense, bien sûr, à la façon dont les nouvelles capacités d'intervention technique peuvent modifier de façon radicale des données biologiques, psychologiques, physiques ou sociales auparavant tenues pour stables. Pour donner un exemple devenu classique, le paradigme de la guerre juste impose deux restrictions dans la conduite de celle-ci (jus in bello): les non-combattants ennemis doivent être épargnés (critère de la discrimination); une opération militaire qui aboutirait à la mort de non-combattants ennemis reste licite à condition ${ }^{38}$ que le nombre de ceux qui sont tués reste proportionné par rapport aux objectifs poursuivis (critère de la proportionnalité). Ces restrictions ont paru impossibles à maintenir dans le cas d'une guerre nucléaire et, de façon générale, d'une guerre où les belligérants utilisent des armes de destruction massive : la base factuelle du paradigme est ici radicalement changée. Parce que les cas paradigmatiques en lesquels s'incarnent les normes sont susceptibles d'être précisés et structurés à la lumière de conditions nouvelles; parce que les bases factuelles à la lumière desquelles les cas paradigmatiques se sont initialement constitués sont susceptibles d'être modifiées de fond en comble, on a donc bien affaire à une variante de constructivisme en matière de normes.

Je voudrais conclure en posant deux questions : cette perpétuelle reconstruction des paradigmes normatifs comporte-t-elle des limites elles mêmes normatives ? Peut-on en dire plus sur cette variante du constructivisme qu'est la nouvelle casuistique ?

La première question est dictée par l'inquiétude suivante : s'il est vrai que des facteurs en provenance de la culture ou de la technique sont susceptibles de présider, en dernière analyse, à l'élaboration des paradigmes, peuton parler d'une autonomie de l'éthique ? R.B. Miller, qui a bien perçu ce que la situation peut avoir d'inconfortable, pour ne pas dire de tragique, répond indirectement à une telle interrogation en dessinant les contours de l'éthique. L'occasion de son analyse est une évaluation de la pornographie violente. Il y voit l'expression d'une sorte de nihilisme moral en ce que la violence qui y est montrée "dénie les conditions pour les limites nécessaire à la structuration d'un univers moral ${ }^{39}$ ». Le nihilisme moral intime le silence parce qu'il défait les conditions mêmes qui rendent possibles un discours éthique. Aussi longtemps, donc, que la reconstruction des paradigmes ne débouche pas sur un tel nihilisme moral, elle semble donc éthiquement acceptable. Mais que

37. Jonsen et Toulmin, The Abuse of Casuistry, p. 318.

38. Il faut également que la mort de ces non-combattants ne soit pas recherchée (elle ne doit pas être un des objectifs de l'opération militaire) et qu'elle ne soit pas un moyen pour atteindre une fin (la mort des non-combattants ennemis ne doit pas pouvoir être comptabilisée au même titre, par exemple, que l'emploi d'une division blindée comme un des moyens mis en oeuvre pour atteindre l'objectif visé).

39. Miller, Casuistry and Modern Ethics, p. 192. 
faut-il préserver pour qu'un tel nihilisme ne s'instaure pas ? Il s'agit de conventions et d'institutions de la vie de tous les jours (everyday life) qui ont pour fonction de promouvoir certains biens : abri (shelter), culture, équité, ordre, santé ${ }^{40}$. R.B. Miller semble donc estimer qu'il existe une moralité implicite de la vie commune, non pas simplement parce qu'elle est là, mais parce qu'en elle se réalisent spontanément des valeurs. Pour l'essentiel, ces analyses semblent partagées, même si elles sont exprimées dans un idiome différent, par A. Jonsen et $\mathrm{S}$. Toulmin : les êtres humains étant ce qu'ils sont, leurs intérêts et leurs buts, pour autant qu'ils sont perpétuellement corrigés et rectifiés par les intérêts et les buts des autres constituent des limites à la plasticité des paradigmes.

Le caractère profondément humien de ces analyses permet de répondre à la seconde question. Mais, en premier lieu, il convient de préciser en quel sens je parle de constructivisme. Le terme, en effet, ne désigne plus seulement la thèse selon laquelle les objets mathématiques ne sont admissibles dans une théorie que s'il existe une méthode permettant de les construire effectivement. Depuis les analyses de John Rawls, il désigne la thèse négative selon laquelle « il n'existe pas quelque chose comme des faits moraux dont les principes adoptés pourraient être une approximation ${ }^{41}$. Chez J. Rawls, le constructivisme comme doctrine positive fait également de la personne un élément d'une procédure de construction destinée à déterminer le contenu des premiers principes de justice. Il s'oppose alors, d'une part, à l'intuitionnisme rationnel selon lequel les concepts moraux fondamentaux ne sont pas susceptibles d'être analysés sans reste en termes de concepts non moraux et pour qui les principes premiers de la morale sont l'objet de propositions immédiatement évidentes ; et, d'autre part, au naturalisme selon lequel les concepts moraux fondamentaux sont formulables en termes de concepts non moraux. De façon immédiatement perceptible, le naturalisme aligne la sphère morale sur la sphère naturelle, indice évident d'une hétéronomie irrecevable dans une perspective kantienne. L'intuitionnisme rationnel peut être interprété comme l'affirmation de l'existence de principes premiers qui « s'imposent en raison de relations objectives dont la nature n'est ni affectée ni déterminée par la conception de la personne ${ }^{42}$; sous cette interprétation, il constitue donc une forme moins immédiatement sensible ou plus insidieuse d'hétéronomie. A la lumière d'une telle classification, la notion même de " constructivisme naturalisé » peut donc sembler une étrangeté. Que peut-on répondre face à une telle perplexité ? Tout d'abord, il semble que les nouveaux casuistes admettraient bel et bien une

40. Ibid, p. 192. La pornographie violente détourne les institutions qui réalisent effectivement ces valeurs (école, hôpital, etc.) en les réduisant au rang de simples décors pour la représentation de fantasmes destructeurs.

41. Rawls, John, « Le Constructivisme kantien » dans Justice et démocratie, tr. fr. de C. Audard, Paris, Seuil, 1993, p. 141.

42. Rawls, « Le Constructivisme kantien », p. 134. 


\section{6 · Philosophiques / Printemps 2001}

partie de la formule de J. Rawls qui a fait l'objet de la note 41 : il n'existe pas de faits moraux que nous parviendrions à formuler de plus en plus exactement par une série d'approximations successives. Mais ils la compléteraient sans doute de la façon suivante : il n'existe pas de faits moraux antérieurs à toute analyse de cas et dont les analyses de cas seraient des descriptions plus ou moins exactes. En ce sens, la nouvelle casuistique constitue bien une version du constructivisme. Reste à établir que ce constructivisme comporte une dimension naturaliste. Le principe de la réponse est assez simple : les nouveaux casuistes ne font pas référence, pour utiliser une distinction kantienne que J. Rawls ne formule pas en ces termes mais qu'il reprend manifestement à son compte et qui est d'ailleurs cruciale chez lui, à une construction pure, mais à une construction empirique. Lorsque J. Rawls fait allusion à des faits moraux, il pense à des faits supposés constituer un "ordre moral antérieur et indépendant " et qui existerait "en dehors de la procédure de construction dans son ensemble ${ }^{43}$, la procédure en question ayant pour but de déterminer des principes de justice. Mais, par définition, de tels principes ne s'appliquent pas aux partenaires dans la position originelle lesquels sont, comme le dit explicitement J. Rawls, motivés par leurs "intérêts supérieurs » ${ }^{44}$, c'est-à-dire par les "intérêts supérieurs de la personnalité morale " ${ }^{45}$. De tels intérêts ne sont pas envisagés par les nouveaux casuistes; sans doute considèrent-ils que l'étude des cas ne saurait être conduite de façon satisfaisante par des individus en proie au tumulte des passions ou motivés par des intérêts partisans et égoistes. Mais les individus auxquels ils pensent ne sont pas des partenaires dans la position originelle et leur préoccupation n'est pas de déterminer des principes qui serviront de conception publique de la justice pour une société bien ordonnée. La casuistique est un art pratiqué par des individus ayant à se prononcer sur des questions en rapport avec la justice sociale. Toutefois, leur ambition n'est pas d'élaborer ou d'appliquer des principes pour décider de ces questions : ils se proposent simplement de construire une solution acceptable à un problème ponctuel. A. Jonsen et $\mathrm{S}$. Toulmin font connaître leur principale critique du modèle déductiviste du raisonnement moral en disant de lui que sa véritable fonction est de garantir une conclusion, des prémisses étant admises, et non de résoudre un dilemme moral (quandary). De façon explicite, ils affirment à propos de l'argumentation en éthique qu'elle a pour objet non pas « des relations théoriques internes à un système de concepts, mais la mise en relation et l'application de ces concepts à l'extérieur, à un monde d'objets concrets et d'états de choses réels ${ }^{46}$. En ce sens, l'importance donnée au modèle déduc-

43. Rawls, «Le Constructivisme kantien », p. 146.

44. Rawls, "Le Constructivisme kantien », p. 146. Egalement : " on les décrit comme n'étant mobilisés que par leurs intérêts supérieurs, ceux qui ont pour objet leurs facultés morales, et par leur souci de réaliser leurs fins ultimes, déterminées quoique inconnues ", "Le Constructivisme kantien ", p. 90.

45. Rawls, " Le Constructivisme kantien », p. 90.

46. Jonsen et Toulmin, The Abuse of Casuistry, p. 327. 
tiviste est l'indice d'une " quête de la certitude » (quest for certainty) que rien ne recommande réellement. A ce propos, il existe un article célèbre de $S$. Toulmin dans lequel il récuse la nécessité d'une telle quête en se réclamant, comme on pouvait s'y attendre, du même John Dewey dont John Rawls fait l'éloge en affirmant que son génie a consisté à " adapter ce qu'il y avait de plus valable dans l'idéalisme de Hegel à une forme de naturalisme spécifique de notre culture ${ }^{47}$.

Le constructivisme dont il est question dans la nouvelle casuistique est le sister-ship du navire de Neurath que l'on reconstruit en haute mer, sans jamais pouvoir le démonter dans un dock et le rebâtir à neuf avec de meilleures pièces. Le pavillon qu'il arbore est bicolore : naturaliste et anti-fondationnaliste.

47. Rawls, "Le Constructivisme kantien ", p. 74. L'article en question s'intitule " The Tyranny of Principles ", The Hastings Center Report, XI, 1981, p. 31-9. La référence à J. Dewey apparaît p. 37. Il n'aura pas échappé au lecteur que lorsqu'il est question de certitude dans le domaine déductiviste, A. Jonsen et $\mathrm{S}$. Toulmin emploient le mot certainty, tandis que c'est le mot certitude qui désigne la certitude propre au modèle casuistique. 